\title{
GRAVITATIONAL RADIATION FROM ACCRETING NEUTRON STARS
}

\author{
BERNARD F. SCHUTZ \\ Max Planck Institute for Gravitational Physics \\ The Albert Einstein Institute \\ Potsdam, Germany \\ E-mail: schutz@aei-potsdam.mpg.de \\ and \\ Department of Physics and Astronomy \\ University of Wales College of Cardiff, Cardiff, U.K.
}

\begin{abstract}
Neutron stars may emit steady gravitational wave signals that will be among the first kinds of gravitational wave signals that the new generation of interferometric detectors will search for. I consider here the possibility that accreting neutron stars may be driven into the steady emission of gravitational waves. I estimate the amplitudes that the waves may have if the accretion takes place at the Eddington limit, such as may happen when a neutron star spirals inside a giant star in the endphase of binary evolution. I consider the computational difficulties of conducting a search for such radiation from known target stars, allowing for the fact that the orbit will not be known from other observations. It seems possible that, with supercomputers, very sensitive searches of a handful of targets may be possible.
\end{abstract}

1. Introduction. Interferometric gravitational wave detectors now under construction in Germany (the GEO600 detector [1]), Italy (VIRGO [2]), and the USA (the two LIGO instruments [3]) will be the first detectors that are expected to be capable of detecting gravitational waves from some anticipated astronomical sources on a reasonable timescale. There are many possible sources [4], but certain features of this first generation of interferometers make them particularly suited to detecting continuous waves from rotating neutron stars. As broadband detectors, they can search a wide range of frequencies above about $100 \mathrm{~Hz}$ (for VIRGO, above $40 \mathrm{~Hz}$ ); and as long-duration projects, they can take coherent data spanning one or more years and thereby build up impressive sensitivity. Moreover, neutron stars are the only sensible target for whichever detector is the first one to operate satisfactorily: continuous waves can be identified reliably by a

1991 Mathematics Subject Classification: Primary 83C35.

The paper is in final form and no version of it will be published elsewhere. 
single detector by tracking their Doppler frequency modulation as the Earth moves; since the source will not go away over a few years, confirmatory observations by other detectors can be made when they reach their design sensitivity. Short-duration gravitational wave bursts, by contrast, can probably not be identified by a single detector operating alone, unless associated with another astronomical observation, such as a neutrino or gamma-ray burst.

Continuous radiation from neutron stars has not received as much attention from theorists as have other sources, such as coalescing neutron star binaries [5]. It has long been recognised that non-axisymmetric irregularities in the crust of a neutron star ("lumps") will radiate. The only observational limits on such radiation come from pulsar spindown: gravitational waves certainly cannot carry away more energy than we infer the pulsar to be losing from its decreasing spin rate. This is a strong upper bound on pulsar amplitude, but is unlikely to be attained in the majority of cases. Moreover, we can only find such bounds for observed radio pulsars: there may be nearby, older neutron stars that are stronger than known ones by virtue of their proximity. The spindown limits for a number of pulsars are within the capabilities for the first generation of detectors, as we discuss below. (See Figure 1.)

Frozen-in lumps are not the only way that neutron stars can radiate: accretion can drive them into non-axisymmetric configurations and power steady radiation with a considerable amplitude.

Wagoner [6] was the first to point this out. Accretion on to neutron stars occurs most obviously in X-ray binary systems, and some of these are potential strong gravitational wave sources [4]. But accretion occurs in other situations, most notably in Thorne-Zytkow objects, which are the stage of evolution of massive X-ray binaries that occurs after the $\mathrm{X}$-ray phase, when the giant companion engulfs the accreting neutron star and smothers its X-radiation. Inside the envelope, however, the neutron star not only continues to accrete, but the rate is believed to go up to near the Eddington rate [7]. Indeed, this is where millisecond pulsars are thought to acquire their high angular momentum.

In this paper I consider the possibility that that such stars may emit detectable radiation. I will consider not only the radiation but the very considerable difficulties that they present if first-generation detectors are to conduct a practical search for them.

2. Radiation from accreting neutron stars. Thorne-Zytkow stars probably look like Be-giants, but they have not received much attention from observers to date, presumably because they are no longer X-ray sources and are difficult to identify optically. But they are more favourable sites for accretion-driven gravitational radiation than standard $\mathrm{X}$-ray binaries. Moreover, they are more numerous: the lifetime of the Thorne-Zytkow phase is perhaps 10 times as long as that of the X-ray binary phase [7], so there could be 10 times as many Be-gaints that contain ne utron stars as there are massive X-ray binaries. The nearest could be as close as $100 \mathrm{pc}$.

Wagoner's mechanism [6] makes a much more definite prediction of gravitational wave amplitudes than does the lumpy-pulsar model, but it applies only to a certain class of neutron stars: accreting stars in binary systems that have been spun up to the first CFS instability point $[8,9]$. Further accretion simply drives this nonaxisymmetric instability 
to the point where gravitational waves carry off all the accreted angular momentum. The amplitude of gravitational waves from a source at a distance $r$, radiating approximately isotropically with a total luminosity $L_{g w}$ and approximately monochromatically at a frequency $f_{g w}$, has an amplitude [10]

$$
h=4 \times 10^{-26}\left(\frac{L_{g w}}{10^{30 \mathrm{~W}}}\right)^{1 / 2}\left(\frac{f_{g w}}{1 \mathrm{kHz}}\right)^{-1}\left(\frac{r}{1 \mathrm{kpc}}\right)^{-1}
$$

In the Wagoner mechanism, the gravitational wave luminosity is a factor $\beta=O(1)$ times the mass-energy accretion rate $\dot{M} c^{2}$. For accretion at a rate of $10^{-10} M_{\odot} \mathrm{yr}^{-1}$, which is typical of X-ray binaries, the expected amplitude is

$$
h=6 \times 10^{-26} \beta^{1 / 2}\left(\frac{\dot{M}}{10^{-10} M_{\odot} \mathrm{yr}^{-1}}\right)^{1 / 2}\left(\frac{f_{g w}}{100 \mathrm{~Hz}}\right)^{-1}\left(\frac{r}{1 \mathrm{kpc}}\right)^{-1}
$$

In Thorne-Zytkow stars the accretion rate is likely to be closer to the Eddington rate for a neutron star, which is about $10^{-8} M_{\odot} \mathrm{yr}^{-1}$.

The frequency $f_{g w}$ is not known ahead of time: it is a property of the unstable mode, and will not equal the neutron star spin frequency. We will return below to the difficulty this makes for a search.

The amplitude of this radiation is larger for lower frequencies, essentially because the energy output is constant. This growth of $h$ with $f^{-1}$ cannot continue to arbitrarily small $f$, however. One limit on $h$ comes from the distortion of the star: the mode amplitude should not be larger than $\delta R \sim R$. A star with an ellipticity $\epsilon=\delta R / R$ that rotates with a frequency $f_{m}$ radiates at $[10]$

$$
h=2 \times 10^{-21} \epsilon\left(\frac{f_{m}}{100 \mathrm{~Hz}}\right)^{2}\left(\frac{r}{1 \mathrm{kpc}}\right)^{-1} .
$$

Setting $\epsilon=1$ shows that the Wagoner mechanism reaches a maximum amplitude at a frequency no lower than 5 or $10 \mathrm{~Hz}$. Modes with frequency less than this (just at the onset of the CFS instability) will grow on the same timescale as the star accretes angular momentum, so that they don't begin radiating away the full accreted angular momentum until the frequency reaches this value. These limits are shown in Figure 1.

The biggest theoretical uncertainty about the existence of such sources of gravitational radiation is the fact that viscosity competes with the CFS instability and can prevent it from having any effect. Viscosity is significantly temperature-dependent. Detailed investigations [11] suggest that there is only a limited range of temperatures inside the neutron star in which the viscosity is small enough for the star to be unstable. A newly formed star may be too hot, and known pulsars are old enough to be too cool to be subject to the instability, even if they were spinning fast enough. It is easy to calculate that a neutron star of radius $10 \mathrm{~km}$ will radiate at the Eddington rate when its temperature is about $3 \times 10^{7} \mathrm{~K}$, so accretion cannot drive the star to a temperature higher than this.

The Newtonian calculations of Lindblom [11] suggest that this is too cold for the instability to operate. But new fully relativistic calculations of mode frequencies [12] show that the CFS instability sets in earlier in realistic models; the calculation of the effect of viscosity in relativistic stars has not yet been done. In any case, our understanding of the 


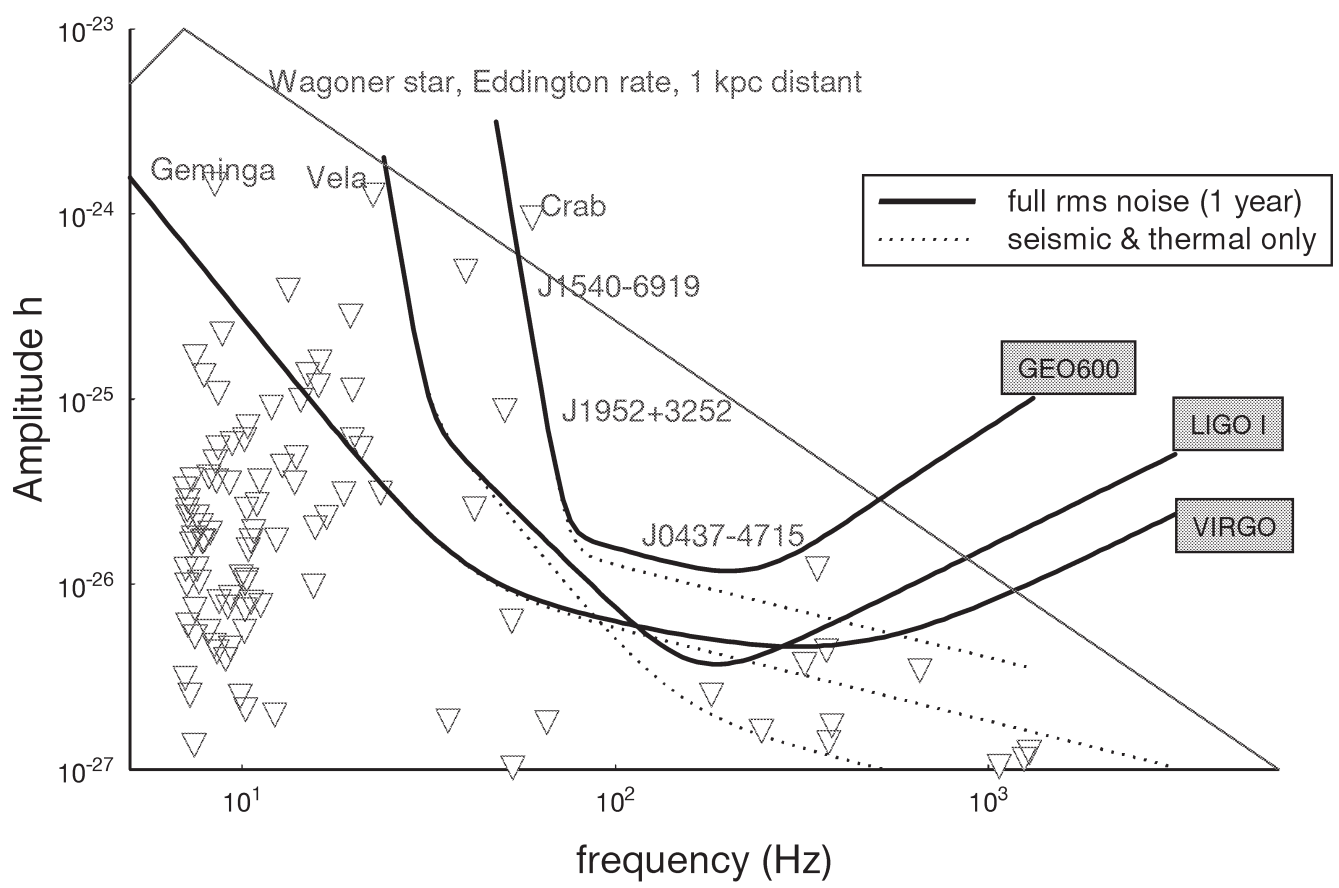

Figure 1: Sensitivity of four interferometers in a 1-year observation, compared to the accretiondriven gravitational wave amplitudes from the Wagoner mechanism.

interiors of neutron stars is supported by very little direct observational evidence, and so it is well worthwhile looking for Wagoner radiation from accreting stars.

3. Searching for accreting stars. Now we turn to the difficulty of reducing data from a gravitational wave detector to identify these objects. In order to get good sensitivity, interferometers need to observe continuously for times of order 1 year, as assumed in Figure 1. During this time, the motion of the interferometer induces important Doppler shifts (phase modulation) and less-important amplitude modulations in the signal. These spread the power from the signal over such a wide bandwidth that the detector noise will hide the signal. Such stars can only be detected by removing the phase modulation. Unfortunately, the pattern of modulation varies greatly over the sky, so a separate reduction must be made for each location. This can be a very demanding task for even the most powerful computers, and it is likely that our sensitivity will ultimately be limited by the computer power rather than observing time.

The first detailed estimates of the difficulties of this detection problem was by the present author [13]. A more extensive treatment in the case of non-accreting field stars will be found in forthcoming paper by Brady, et al [14]. That paper concludes that a search of the whole sky for a radiating neutron star that is spinning down but is not in a binary orbit can be done with a teraflop computer only for data sets of a few days in length. To find an accreting star, which must have additional phase modulation from its orbital motion around the companion that supplies the accreting gas, would be hopeless. 
However, one can contemplate a targeted search, where specific positions are searched for orbiting stars. Since the timescale for the completion of the Thorne-Zytkow process may be $10^{5} \mathrm{yr}$ or more, the time-scale for the intrinsic frequency of the star may be much longer than assumed by Brady, et al, and this again reduces the parameter space that must be searched. Moreover, the orbital of the star can be taken to be circular, if it is inside the envelope of the companion: eccentricity will rapidly dissipate. Here we make some preliminary estimates of the difficulty of a search.

Suppose that we have a data set lasting $10^{7} \mathrm{~s}$. Its frequency resolution is $10^{-7} \mathrm{~Hz}$, and we are looking for a star with an unknown frequency that may be as high as $10^{3} \mathrm{~Hz}$.

The Earth changes its velocity by of order $10^{-4} c$ during this time, inducing a Doppler shift of order $0.1 \mathrm{~Hz}$ in the pulsar signal. This is $10^{6}$ frequency resolution elements, and it shows that the angular resolution of an observation of a star will be of order $10^{-6} \mathrm{rad}$, or of order one arcsecond. If the position of a target star is known to this accuracy, then one does not have to search extensive regions of the sky.

If the star is spinning up on a timescale of $10^{12} \mathrm{~s}$, then during the observation period it will have changed its frequency by no more than one part in $10^{5}$, or $10^{-2} \mathrm{~Hz}$. The second derivative should be smaller, of order the square of this dimensionless ratio, or one part in $10^{10}$. This would be just at the frequency resolution of the observation, so we can neglect it: a linear model for frequency change is adequate here. The number of distinguishable first derivatives of the frequency that we need to include as parameters is roughly the same as the number of distinguishable final frequencies the signal can reach in the observing time, up to our assumed maximum rate. This is $10^{5}$. Now we estimate the number of orbital parameters we need. If we assume a circular orbit, then we need consider only orbital radius, period and inclination. The inclination of the orbit only affects the velocity of the star along the line of sight: an orbit viewed face-on has no modulation, while one viewed in the plane experiences the full orbital modulation. Thus, we can represent these three intrinsic parameters by two degrees of freedom in the signal model: the modulation frequency (orbital frequency $f_{\text {orb }}$ ) and the modulation amplitude (determined by the radius of the orbit projected onto the line of sight, which I will call $\left.R_{e f f}\right)$.

The form of the signal's Fourier transform is not hard to guess. (It can be found in any standard text on communications: frequency modulation of this type is the basis of FM radio transmission.) The modulation is periodic with the orbital frequency $f_{\text {orb }}$, so the signal looks like a Fourier series with spikes of power separated by this distance. The power in the signal is spread over a range around the pulsar frequency $f_{p}$ that is equal to the maximum change in the frequency of the signal due to the modulation, which is given by $\Delta f_{p} / f_{p}=2 \pi R_{e f f} f_{\text {orb }} / c$. The number of power spikes in the modulated signal is $\Delta f_{p} / f_{\text {orb }}=2 \pi f_{p} R_{e f f} / c=2 \pi R_{e f f} / \lambda_{g w}$, where $\lambda_{g w}$ is the gravitational wavelength. Now we estimate the size of these numbers and the accuracy with which they can be determined.

The frequency of the modulation $f_{\text {orb }}$ goes at least as high as the orbital frequency of the Hulse-Taylor pulsar, since that was presumably formed by the process we are considering, but it may go higher if some recent binary evolution models are correct. I will assume we will search all modulation frequencies up to $10^{-3} \mathrm{~Hz}$, which is higher than 
that of any known binary. In the Fourier transform of the received signal, this frequency can be measured by finding the positions of the power spikes. This can presumably be done with an accuracy equal to the frequency resolution of the observation, $10^{-7} \mathrm{~Hz}$. Therefore, we need $10^{4}$ possible parameter values for the orbital frequency.

The amplitude of the modulation is determined by (see above) the number of wavelengths of the gravitational wave $\lambda_{g w}$ that fit across the binary orbit $R_{e f f}$. This orbit is of order a few solar radii, say $10^{10} \mathrm{~m}$ at most. The wavelength of the gravitational wave is no smaller than $3 \times 10^{5} \mathrm{~m}$, so there are no more than $3 \times 10^{4}$ wavelengths across the orbit. Allowing for the factor of $2 \pi$ in our formula for the number of power spikes, we see that we need a number of parameters that is about $10^{5}$ to span the possible modulation amplitudes.

The product of all these independent parameters is $10^{5} \times 10^{4} \times 10^{5}=10^{14}$. For a full search, presumably something like a Fourier transform has to be done for each parameter, although maybe some sort of "stepping" algorithm such as may be used for a position search [13] could reduce the work to of order $N$ calculations per parameter value rather than $N \log N$. For our set of $N=10^{10}$ data points, we thus have of order $10^{24}$ calculations to perform for each search. For a teraflop computer this will still take over 30 thousand years!

The cost of this search can, of course, be reduced by taking more restrictive assumptions for the orbital parameters, but this may not be desirable astrophysically. It may also be possible to reduce the cost of the search by two-stage methods, where a first stage eliminates much of the data and focuses on only a restricted set that needs a full analysis. This is possible in our case because, to be detectable, a signal will have to have a high signal-to-noise ratio, of order $10 \sigma$, in order to discriminate against expected accidentals in the $10^{24}$ different output numbers (over all the parameters and frequencies). So a search does not have to go down to $1 \sigma$. The first stage of a two-stage search could be crude and only recognize the signal at, say, $3 \sigma$; a threshold at this level would then lead one to the second stage, where the signal and a number of accidentals would have to be followed up, but only in a narrower range of parameters that were indicated by the results of the first stage.

One such approximate method is a hierarchical method, such as has been discussed elsewhere for the all-sky problem. The number of parameters counted above scales as $T_{\text {obs }}^{2}$, and the number of data points also scales as $T_{o b s}$. So the computing cost is proportional to $T_{o b s}^{3}$. By reducing $T_{o b s}$ to about 1 day, the computing time on a teraflop computer comes down also to about 1 day. This is an improvement, but it reduces the sensitivity of the observation by a factor of 10. However, by "stacking" (adding) power spectra from successive days, one can recover a factor of about 3 , so that the signal would have an amplitude of $3 \sigma$ in the stacked transforms. Having identified the signal, then it could be filtered for over a narrow range of parameters, restoring it to the full sensitivity.

Another approximation method might be to filter the signal's power spectrum for a specified number of power spikes, eliminating the modulation amplitude as a parameter. This reduces the parameter space to a manageable amount: such a calculation could be done for $10^{7} \mathrm{~s}$ of data on a teraflop computer in $10^{7} \mathrm{~s}$. But it would have lower signal-tonoise, and I know of no estimates of how it would work in practice. 
One can hope, therefore, that some variant on one of these methods might allow one to search a few targets at nearly optimum sensitivity.

4. Conclusion. We have seen that it would be interesting to search for signals from accreting neutron stars, but these must allow for the modulation of the signal produced by the orbital motion of the star. If the searches can be done in a reasonable amount of time on a fast computer, then Figure 1 suggests different strategies for different detectors. The GEO600 detector should target only the nearest Be-giants. LIGO I should look at stars out to several hundred pc. VIRGO has the best chance of the first-generation detectors, and should target all Be-giants within $1 \mathrm{kpc}$.

Many questions discussed here need further investigation. Given the large number of Thorne-Zytkow objects that may exist in the Galaxy, and the fact that the first interferometer to reach an interesting sensitivity will almost certainly perform a search for continuous waves as a first priority, a neutron star in such a system may well be the strongest continuous source of gravitational radiation in the sky. We should not lose the opportunity to detect it.

\section{References}

[1] K. Danzmann, "GEO 600 - A 600-m Laser Interferometric Gravitational Wave Antenna", in E. Coccia, G. Pizzella, F. Ronga, eds., Gravitational Wave Experiments, (World Scientific, Singapore, 1995), p. 100-111.

[2] C. N. Man et al., "The Virgo Project: a Progress Report", in D. Blair, M.J. Buckingham, eds., The Fifth Marcel Grossmann Meeting, (World Scientific, Singapore, 1989), p. 17871801.

[3] A. Abramovici et al., "LIGO: The Laser Interferometer Gravitational-Wave Observatory", Science, 256, 325-333 (1992).

[4] K. S. Thorne, "Gravitational Radiation", in S.W. Hawking, W. Israel, eds., 300 Years of Gravitation, (Cambridge University Press, Cambridge, 1987), p. 330-458.

[5] C. Cutler et al., Phys. Rev. Lett., 70, 1984 (1993).

[6] R. V. Wagoner, Astrophys. J., 278, 345 (1984).

[7] E. P. J. Van den Heuvel, and D. Bhattacharya, Phys. Rep., 303, 1-121 (19??).

[8] S. Chandrasekhar, Phys. Rev. Lett., 24, 611 (1970).

[9] J. L. Friedman, B. F. Schutz, "Secular Instability of Rotating Newtonian Stars", Astrophys. J., 222, 281-296 (1978).

[10] C. W. Misner, K. S. Thorne, J. L. Wheeler, Gravitation, (Freeman, San Francisco, 1973).

[11] L. Lindblom and G. Mendell, Astrophys. J., 444, 804-809 (1995).

[12] N. Stergioulas, The Structure and Stability of Rotating Relativistic Stars (PhD Thesis, University of Wisconsin-Milwaukee, 1996).

[13] B. F. Schutz, "Data Processing Analysis and Storage for Interferometric Antennas", in D.G. Blair, ed., The Detection of Gravitational Waves, (Cambridge University Press, Cambridge England, 1991), p. 406-452.

[14] P. Brady, T. Creighton, C. Cutler, and B. F. Schutz, "Searching for periodic sources with LIGO", submitted for publication (gr-qc9702050). 